\title{
Gastric Adenocarcinoma of the Fundic Gland Type after Endoscopic Therapy for Metachronous Gastric Cancer
}

\author{
Hitoshi Kino ${ }^{1}$, Masakazu Nakano ${ }^{1}$, Akira Kanamori ${ }^{1}$, Tsunehiro Suzuki ${ }^{1}$, Yoshihito Kaneko ${ }^{1}$, \\ Chieko Tsuchida ${ }^{1}$, Kouhei Tsuchida ${ }^{1}$, Keiichi Tominaga ${ }^{1}$, Takako Sasai ${ }^{1}$, \\ Hidetsugu Yamagishi ${ }^{2}$, Yasuo Imai ${ }^{2}$ and Hideyuki Hiraishi ${ }^{1}$
}

\begin{abstract}
:
A 78-year-old man underwent endoscopic submucosal dissection (ESD) for early gastric adenocarcinoma twice in 2009 and 2014. Between the procedures, he successfully completed Helicobacter pylori eradication therapy. In May 2015, upper endoscopy screening showed two elevated lesions on the gastric fundus, and en bloc resection by ESD was performed. We histopathologically diagnosed the patient to have gastric adenocarcinoma of the fundic gland type. In this case, the two lesions of gastric adenocarcinoma of the fundic gland type multifocally developed after ESD for metachronous gastric tubular adenocarcinoma. Furthermore, they appeared in the gastric fundus, where atrophy had been improved due to eradication therapy.
\end{abstract}

Key words: gastric adenocarcinoma of fundic gland type, Helicobacter pylori, endoscopic submucosal dissection

(Intern Med 57: 795-800, 2018)

(DOI: 10.2169/internalmedicine.9359-17)

\section{Introduction}

Ueyama et al. recently proposed a new histological type of gastric cancer manifesting with differentiation towards the fundic gland, called gastric adenocarcinoma of the fundic gland type (1) (GA-FG). This type of gastric carcinoma commonly occurs in the upper portion of the stomach in the elderly and is derived from the deep part of the normal fundic gland without atrophy; furthermore, even small lesions are frequently reported to have invasion to the submucosa. Although this gastric adenocarcinoma is generally thought to develop in non-atrophic mucosa in Helicobacter pylorinegative patients, it has been reported to occur in nonatrophic mucosa even in patients with a history of eradication and those positive for $H$. pylori (2).

We herein report our experience of two lesions of gastric adenocarcinoma of the fundic gland type that developed after endoscopic submucosal dissection (ESD) for metachronous adenocarcinoma of usual type in a patient who had re- ceived eradication five years earlier.

\section{Case Report}

A 78-year-old man underwent ESD for early gastric tubular adenocarcinoma on the lesser curvature of the middle corpus in September 2009, and curative resection was achieved. At the time of endoscopic treatment, the lesion was characterized as atrophic gastritis of grade O-I according to the Kimura/Takemoto classification (3) (Fig. 1a), and he was positive for anti- $H$. pylori immunoglobulin (Ig) G antibody (serum level, $23 \mathrm{U} / \mathrm{mL}$ ). He therefore received $H$. pylori eradication therapy (lansoprazole, amoxicillin, and clarithromycin) in January 2010, and a urea breath test confirmed successful eradication two months later.

Although he was followed up by annual esophagogastroduodenoscopy (EGD), metachronous early gastric tubular adenocarcinoma was detected on the lesser curvature of the lower corpus in September 2014. ESD was performed, resulting in curative resection.

${ }^{1}$ Department of Gastroenterology, Dokkyo Medical University, Japan and ${ }^{2}$ Department of Diagnotic Pathology, Dokkyo Medical University, Japan

Received: April 17, 2017; Accepted: July 14, 2017; Advance Publication by J-STAGE: December 8, 2017

Correspondence to Dr. Hitoshi Kino, jin58fenrir@yahoo.co.jp 

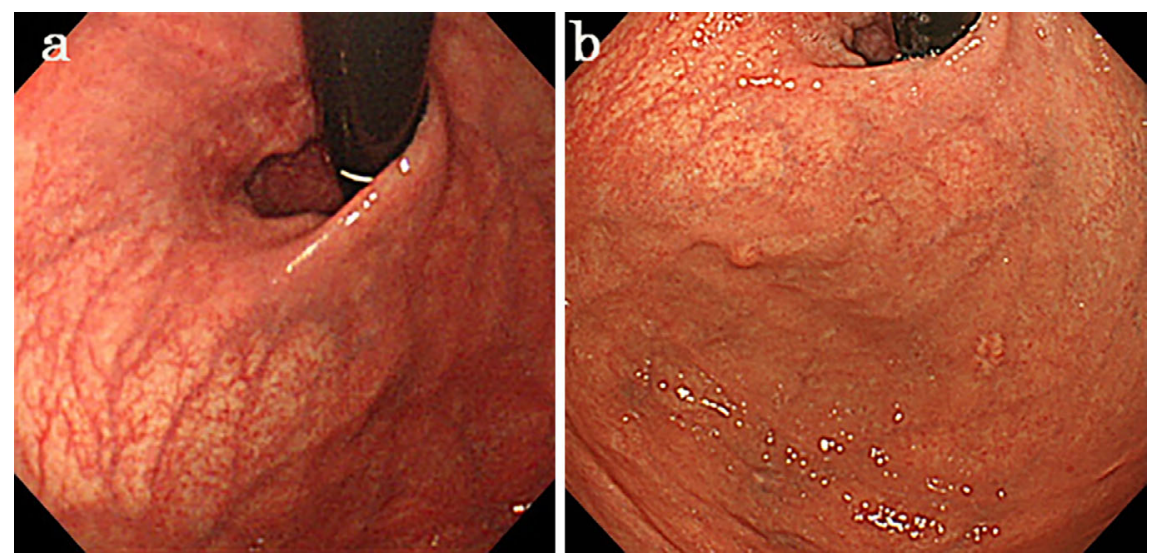

Figure 1. The gastric mucosa shows extensive atrophy before Helicobacter pylori eradication (a). Five years after $\boldsymbol{H}$. pylori eradication, atrophy of the gastric mucosa improved, but two new lesions were detected in the gastric fundus.
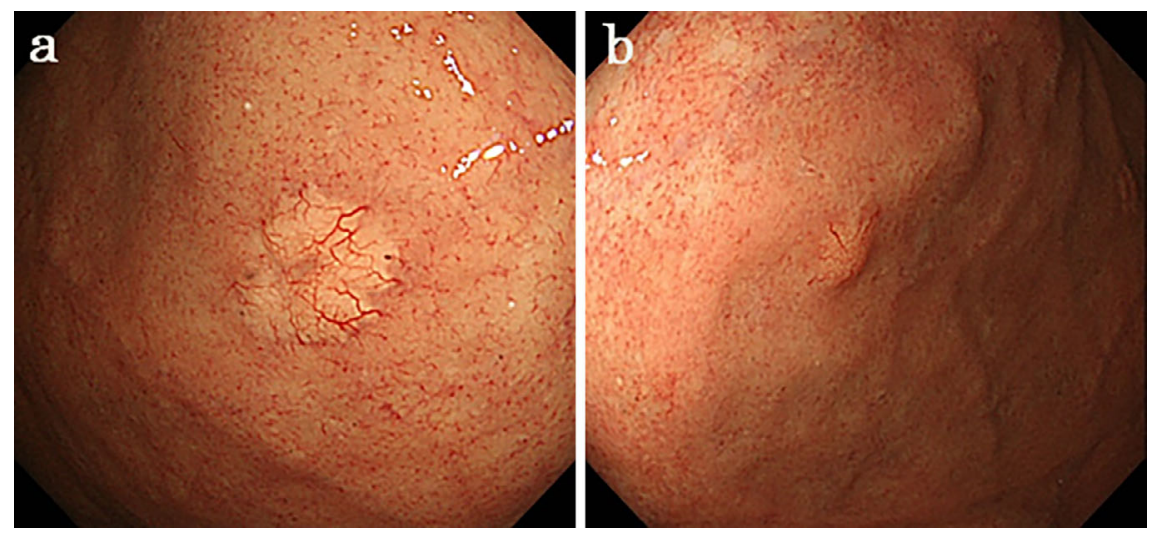

Figure 2. Yellowish protruding lesions are observed on the anterior (a: first lesion) and posterior (b: second lesion) walls of the gastric fundus.

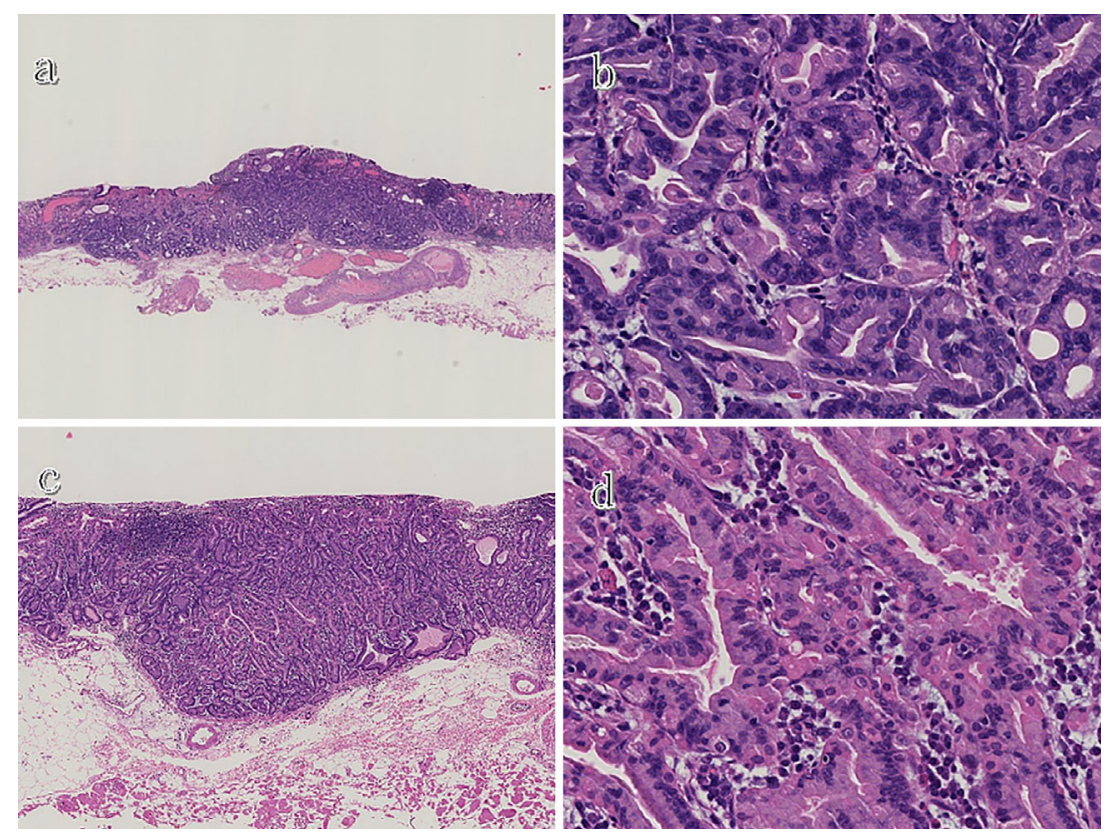

Figure 3. A histological examination of the resected specimens (Hematoxylin and Eosin staining). Low magnification of the first lesion shows that its main portion is located in the deep layer of the lamina propria and that it has slightly infiltrated into the submucosa (a), whereas high magnification reveals that both lesions consist of fundic chief cell-like basophilic columnar cells (b and d). Low magnification of the second lesion shows that, although its main portion is located in the deep layer of the lamina propria, the entire lesion is confined within the mucosa (c). 

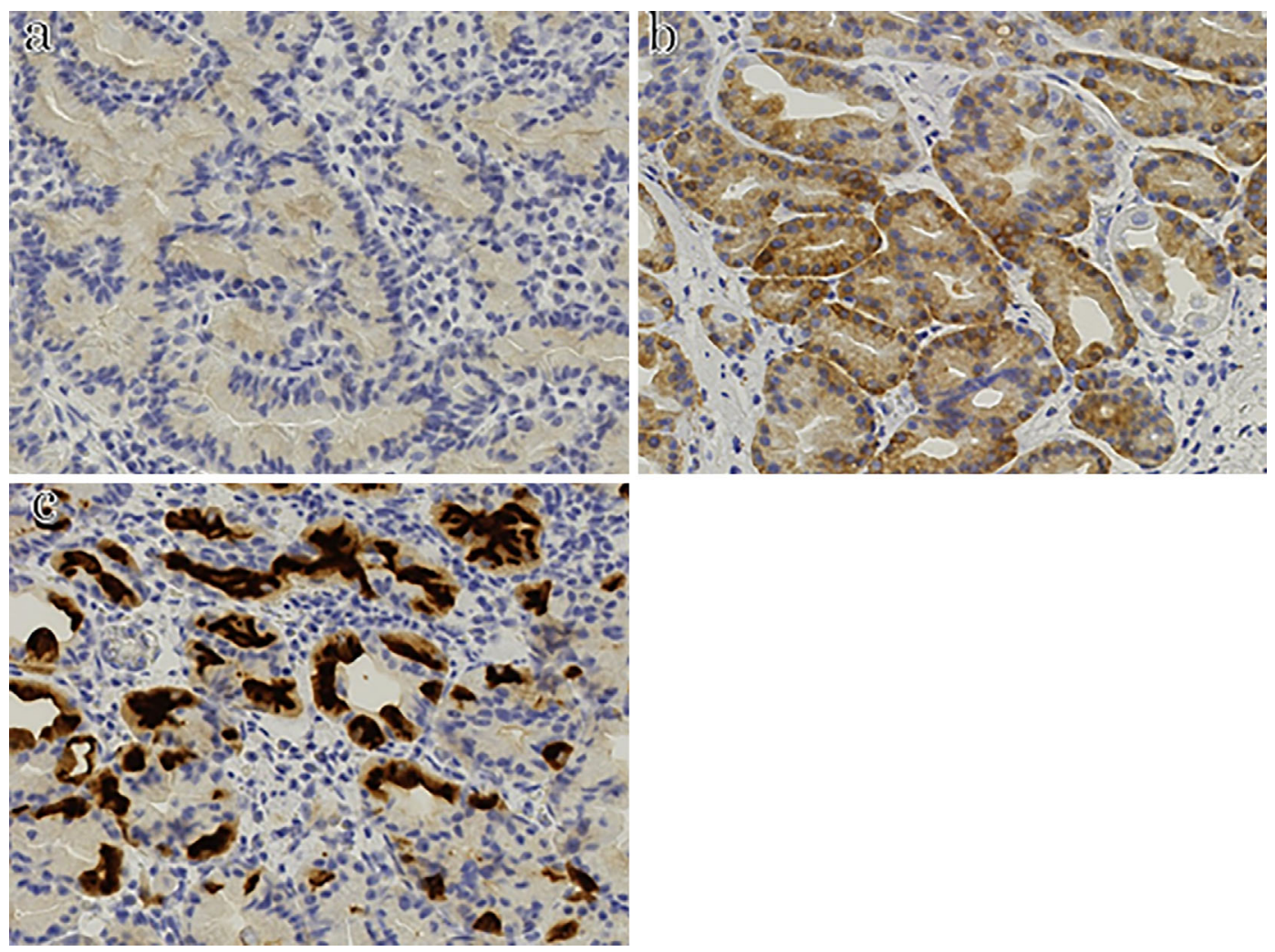

Figure 4. Immunostaining shows that both lesions are positive for pepsinogen I (a), MUC6 (b), and $\mathrm{H}^{+} / \mathrm{K}^{+}$-ATPase (c).
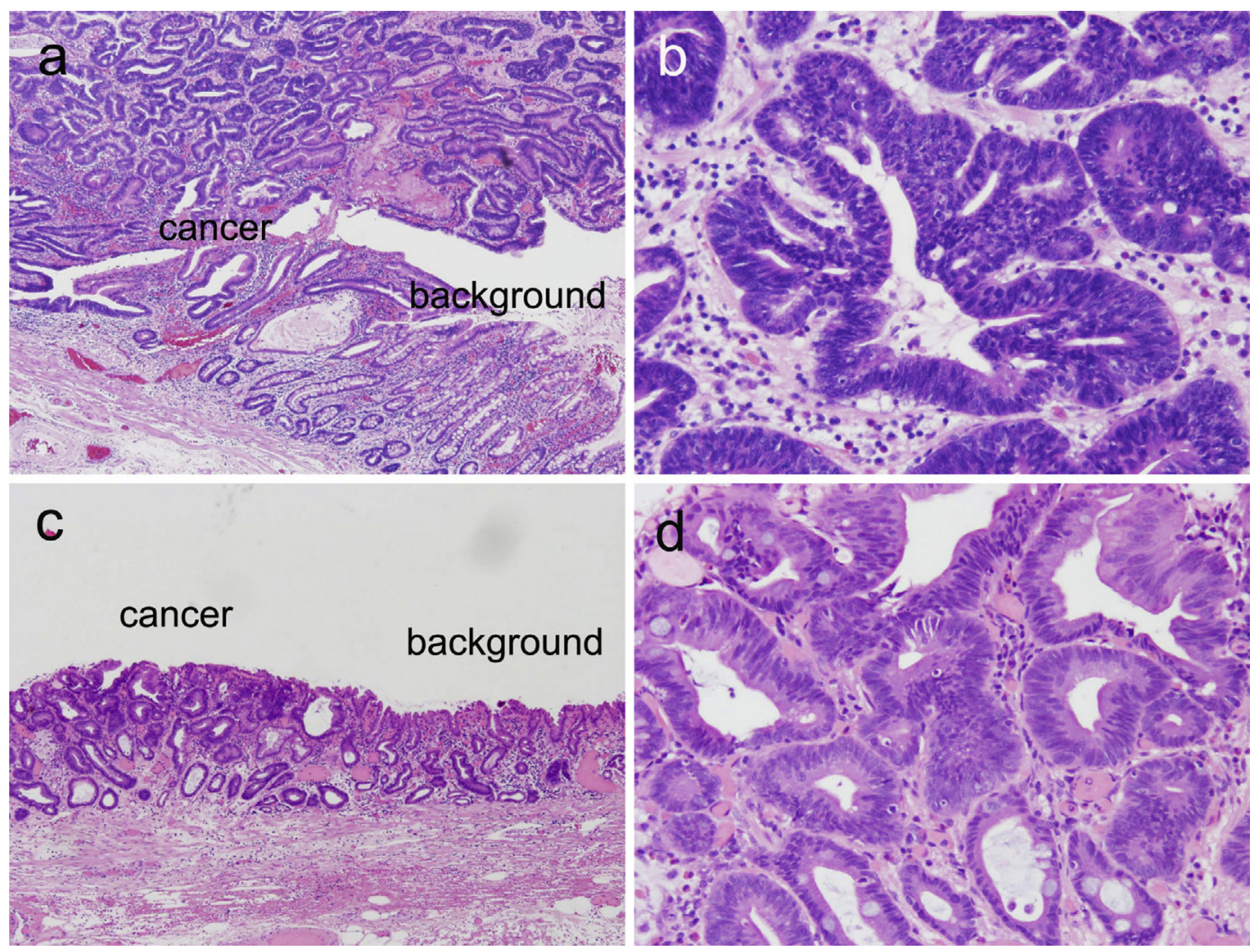

Figure 5. Histology of gastric cancers resected in 2009 and 2014. The border between the cancer and background non-neoplastic mucosa of the cancers resected in 2009 (a) and 2014 (c) [Hematoxylin and Eosin (H\&E) staining, $\times 4$ ]. Higher-power view of the cancers resected in 2009 (b) and 2014 (d) (H\&E staining, $\times 20)$. 

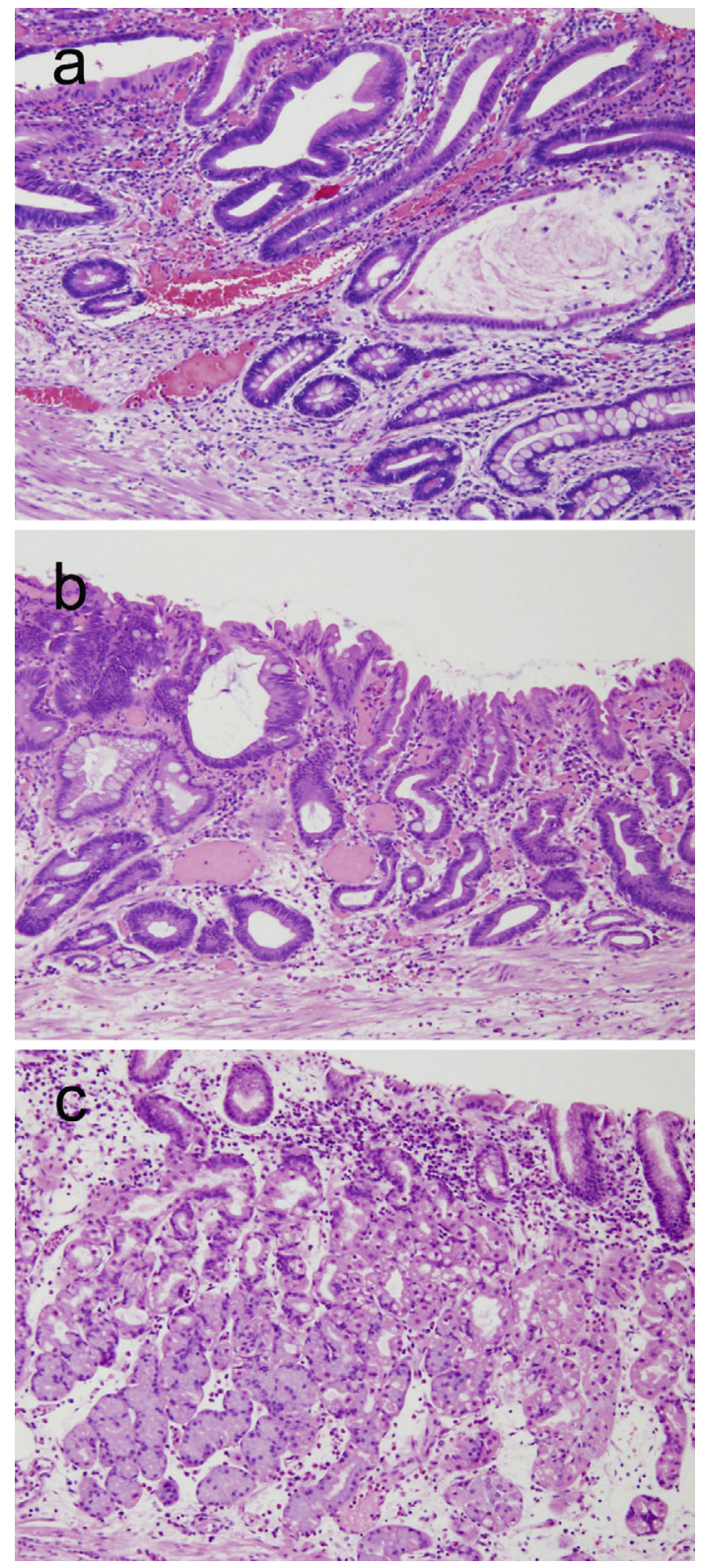

Figure 6. Histology of the background gastric mucosa adjacent to the cancers resected in 2009 (a), 2014 (b), and 2015 (c) (Hematoxylin and Eosin staining, $\times 10$ ).

In March 2015, follow-up EGD revealed two yellowishwhite submucosal tumor (SMT)-like protrusions measuring 4 and $7 \mathrm{~mm}$ in size, with markedly dilated blood vessels on the anterior and posterior walls of the gastric fundus (Fig. 1b, 2a and b). The background mucosa of the lesions was endoscopically and pathologically free of mucosal atrophy because of eradication.

A pathological examination of the biopsy specimens revealed a fundic gland with irregularly arranged ducts with slightly swollen nuclei, while the specimens were immunohistologically positive for MUC6, pepsinogen I, and hydrogen/potassium-adenosine triphosphatase $\left(\mathrm{H}^{+} / \mathrm{K}^{+}\right.$-ATPase $)$. GA-FG was suspected. Blood test results, including tumor markers, were normal, and the patient was negative for antiH. pylori $\mathrm{IgG}$ antibody with a level of $3 \mathrm{U} / \mathrm{mL}$.

We performed ESD for two gastric adenocarcinoma le- sions (fundic gland type) in the gastric fundus and were able to resect each of them en bloc. The pathological findings revealed that the main portions of the lesions were located in the deep layer of the lamina propria and were composed of fundic chief cell-like basophilic columnar cells (Fig. 3a-d). Although one of the lesions had minimal invasion to the submucosa (120 $\mu \mathrm{m}$ from the muscularis mucosae) (Fig. 3a), neither lymphatic nor venous invasion was recognized. The horizontal and vertical margins were negative.

Immunostaining showed that the lesions were negative for MUC2, MUC5AC, and chromogranin A and positive for MUC6, pepsinogen I, and $\mathrm{H}^{+} / \mathrm{K}^{+}$-ATPase (Fig. 4).

Based on these findings, one lesion was diagnosed as a protruding gastric adenocarcinoma of the fundic gland type (chief cell predominant type, GA-FG-CCP) confined within the mucosal layer, and the other was diagnosed as a protruding GA-FG-CCP with minimal invasion to the submucosa $(120 \mu \mathrm{m})$. Both lesions were determined to have achieved a relative curative resection. EGD performed one year after ESD showed no evidence of recurrence in the stomach, and computed tomography also showed no evidence of metastasis to other organs.

\section{Discussion}

Since Ueyama et al. proposed the concept of GA-FG-CCP in 2010 (1), multiple cases of this disease have been reported. It commonly occurs in the upper third of the stomach in the elderly and is derived from the deep part of the normal fundic gland without atrophy, and even small lesions are frequently reported to have invasion to the submucosa. However, because of the low cell proliferative activity and reports of only a few cases of recurrence and metastasis, this type of gastric adenocarcinoma is considered to be a lowgrade malignancy with a favorable prognosis (1). At our hospital, we have also encountered tumors of a similar histological type.

Ueyama et al. broadly divided the endoscopic findings of gastric adenocarcinoma of the fundic gland type into the SMT shape and the flat or depressed type. Based on the most common endoscopic findings, they reported that this disease is characterized by the SMT shape, whitish color, dilated blood vessels with branching architecture, and background mucosa without atrophy (4). Both lesions in our case exhibited similar characteristics.

Because $H$. pylori infection is not involved in the development of GA-FG, it is generally thought to be derived from non-atrophic mucosa (4). In our case, endoscopic procedures had been performed to treat gastric adenocarcinoma of usual type developing from atrophic gastritis, but EGD performed in September 2009 revealed atrophic mucosa extending beyond the gastric cardia and reaching the gastric fundus, which was not histologically assessed. However, the background mucosa of GA-FG that developed in the gastric fundus after eradication was endoscopically and pathologically free of mucosal atrophy. While atrophy is known to be 


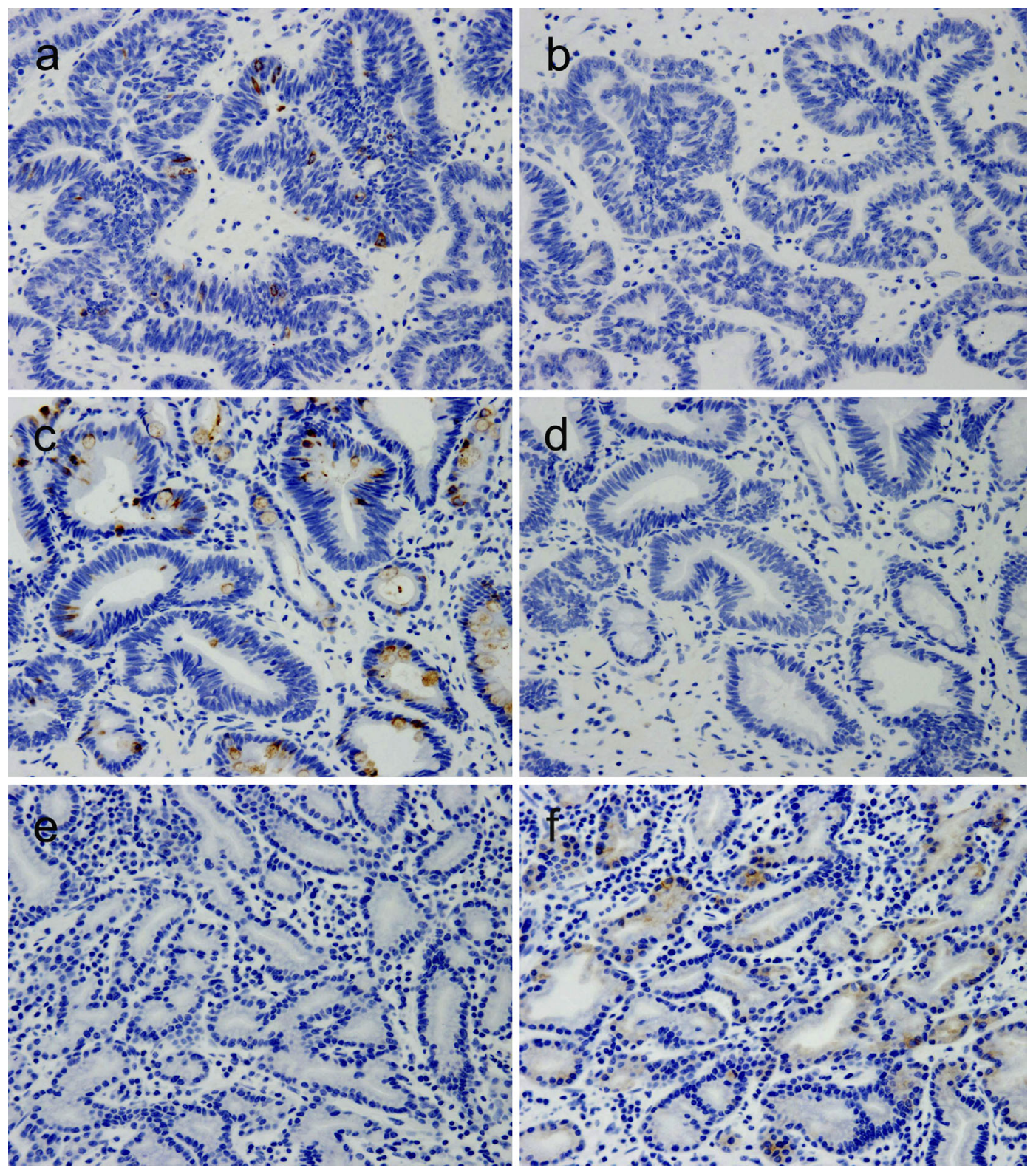

Figure 7. The mucin expression status. The MUC2 expression in cancers resected in 2009 (a), 2014 (c), and 2015 (e), and the MUC6 expression in gastric cancers resected in 2009 (b), 2014 (d), and 2015 (f) (Hematoxylin and Eosin staining, $\times 10)$.

histologically improved after eradication $(5,6)$, we assumed that, in our case, GA-FG occurred in the gastric fundus where mucosal atrophy had been improved by eradication.

The gastric cancers resected in 2009 and 2014 were found to have developed in the intestinal metaplasic mucosa, while those resected in 2015 were found to have developed in the non-atrophic gastric mucosa (Fig. 5, 6). H. pylori infection is known to cause atrophy and intestinal metaplasia of the gastric mucosa, which can be a precursor of intestinal-type gastric cancer (7). Indeed, MUC2 expression, but not MUC6 expression, was observed in these two cancers, demonstrating an intestinal phenotype (Fig. 7). However, the GA-FGCCPs resected in 2015 both developed in the non-atrophic mucosa and demonstrated gastric phenotype with the expression of MUC6 but not MUC2 (Fig. 6, 7). Reportedly, the activation of $\beta$-catenin signaling is a central component in the regulation of pre-malignant epithelial responses to $H$. pylori (8). To demonstrate this, we performed $\beta$-catenin immunostaining in the present four cancers but observed no nuclear accumulation of $\beta$-catenin in any of the present cases (data not shown). The GA-FG-CCPs resected in 2015 occurred in non-atrophic gastric mucosa and bear a gastric phenotype, suggesting that their origin is unrelated to $H$. $p y$ lori infection.

Although the oncogenic mechanism of GA-FG has not been elucidated, it has been reported to be associated with mutations of the $\mathrm{Wnt} / \beta$-catenin signaling pathway and GNAS and KRAS (9-12). In the present case, the two lesions of the conventional gastric cancer and the two lesions of GA-FG developed multifocally, suggesting that these types of gastric cancer may share common risk factors. More clinical data are needed to confirm the common risk factors. 
After ESD for early gastric adenocarcinoma and eradication, patients should be carefully followed up for metachronous lesions of not only gastric adenocarcinoma of usual type but also GA-FG.

The authors state that they have no Conflict of Interest (COI).

\section{References}

1. Ueyama H, Yao T, Nakashima Y, et al. Gastric adenocarcinoma of fundic gland type (chief cell predominant type): proposal for a new entity of gastric adenocarcinoma. Am J Surg Pathol 34: 609619, 2010.

2. Chiba T, Kato K, Masuda T, et al. Clinicopathological features of gastric adenocarcinoma of the fundic gland (chief cell predominant type) by retrospective and prospective analyses of endoscopic findings. Dig Endoscopy 28: 722-730, 2010.

3. Kimura K, Takemoto T. An endoscopic recognition of the atrophic border and its significance in chronic gastritis. Endoscopy 1: 8796, 1969.

4. Ueyama H, Matsunmoto K, Nagahara A, Hayashi T, Yao T, Watanabe S. Gastric adenocarcinoma of fundic gland type (chief cell predominant type). Endoscopy 46: 153-157, 2014.

5. Vannella L, Lahner E, Bordi C, et al. Reversal of atrophic body gastrirtis after H. pylori eradication at long-term follow-up. Dig
Liver Dis 43: 295-299, 2011.

6. Kodama M, Murakami K, Okimoto T, et al. Ten-year prospective follow-up of histological changes at five points on the gastric mucosa as recommended by the updated Sydney system after Helicobacter pylori eradication. J Gastroenterol 47: 394-403, 2012.

7. Correa P, Piazuelo MB. Helicobacter pylori infection and gastric adenocarcinoma. US Gastroenterol Hepatol Rev 7: 59-64, 2011.

8. Polk DB, Peek RM Jr. Helicobacter pylori: gastric cancer and beyond. Nat Rev Cancer 10: 403-414, 2010.

9. Lee SY, Saito T, Mitomi H, et al. Mutation spectrum in the Wnt/ $\beta$-catenin signaling pathway in gastric fundic gland associated neoplasms/polyps. Virchows Arch 467: 27-38, 2015.

10. Hidaka Y, Mitomi H, Saito T, et al. Alteration in the Wnt/ $\beta$-catenin signaling pathway in gastric neoplasias of fundic gland (chief cell predominant) type. Hum Pathol 44: 2438-2448, 2013.

11. Kushima R, Sekine S, Matsubara A, Taniguchi H, Ikegami M, Tsuda H. Gastric adenocarcinoma of the fundic gland type shares common genetic and phenotypic features with pyloric gland adenoma. Pathol Int 63: 318-325, 2013.

12. Matsubara A, Sekine S, Kushima R, et al. Frequent GNAS and KRAS mutations in pyloric gland adenoma of the stomach and duodenum. J Pathol 229: 579-587, 2013.

The Internal Medicine is an Open Access article distributed under the Creative Commons Attribution-NonCommercial-NoDerivatives 4.0 International License. To view the details of this license, please visit (https://creativecommons.org/licenses/ by-nc-nd/4.0/).

\footnotetext{
(C) 2018 The Japanese Society of Internal Medicine Intern Med 57: 795-800, 2018
} 